\title{
The Immune Network System with Chaotic Behavior
}

\author{
Algis GARLIAUSKAS \\ Institute of Mathematics and Informatics \\ Akademijos 4, LT-06883, Vilnius, Lithuania \\ e-mail: galgis_1@ktl.mii.lt
}

\begin{abstract}
In this paper the immune network system was presented by the sequence of species with new immunological components allowing more plausible to reflect the immune response processes. The mathematical model oriented to the describing of the more realistic immune processes in the dynamics allowed to organize the computational experiment in the first to demonstrate a possible very complex behavior including chaotic regimes. The results of modeling of dynamic immunological processes with chaotic behavior are represented and considered.
\end{abstract}

Keywords: immune network system, dynamics, mathematical model, chaotic behavior, bifurcation diagram.

\section{Introduction}

The immune system based on an immune response to different endogenic active selfreplicating antigens such as virus, bacteria or foreign cells represents an arch-complex biological system, researching of which is denoted by numerical theoretical and experimental works in the world. Note, that the immune system has much similarities to the neural system. Both systems display dichotomies and dualism. The cells of two systems may receive signals as well as transmit they. The signals can be either excitatory or inhibitory. In human body, there are about $10^{11}$ neurons and about $10^{12}$ of the immune system cells - lymphocytes. Latter did not connect by strict fibres in order to form a network though (as we will show below) some prepositions to form a network take place. The neural networks enable much more present by networking structure. Both systems develop from experience and build up a memory and are deposited in persistent network, which cannot be transmitted to the offsprings.

For immune system, the clonal theory proposed by Burnet (1959) plays an important role in satisfactory explanation for the formation of antibody specific to antigen that is infected into binds to the receptors of the lymphocytes and macrophages. Latter attracts B lymphocytes which sensitize the proliferation of plasma-cells and produce antibodies. This immunological postulate is confirmed by a very large amount of experimental works.

Today the experimental and theoretical immunology accumulate the crucial material about fundamental results concern to mutual activities between antigens and antibodies 
on the different level of details from microscopical to intercellular genetic cases. These results allow to come up a building of mathematical models of the immune processes.

One of the first direction to the construction of the immune mathematical model is the work (Hege and Cole, 1966) in which it has built the equation, describing the change of amount of circulating antibodies dependent on the number of the plasma-cells the same specific. After that Jilek (1971a, 1971b) proposed the simple mathematical model and the probability approach to the immunocompetant cells with antigen simulated by Monte-Carlo process of clone forming from one $B$ cell.

Bell $(1970,1971,1973)$ has built a mathematical model of humoral immune reaction based on hypothesis of the clonal selection theory Burnet (1959). Mohler et al. (1978) suggested the model for presentation and analysis of the antibody production and cooperation between $T$ and $B$ systems of the immune response. The wide class of dynamic nonlinear models with dilation have been analyzed by Marchuk $(1978,1980)$.

In 1975 Richter (1975) and Hoffmann (1975) have proposed original immune models based on some grounds of the network theory of the immune system Jerne (1974). Richter and Hoffmann have done a set of assumptions concerning to the interactions in the network with low zone tolerance of immune response.

Apart dynamics of the immune response processes analyzed by Richter (1975), Hoffmann (1975), Marchuk (1980), Prikrilova et al. (1984) and others, Bell (1970) set the limit circles with self-content states, attractive focal points or saddle points. But in these all works no attempts to see to the immunological systems from position of a selforganization in part or synergetics in common case. We try to represent the immune system as a massive multicomponent nonlinear network with clonal structure and complex interconnected subblocks both an humoral and cellular components of the general immune system.

According to Stepney (2003) or Nicolis and Prigogin (1979) self-organizing systems have bifurcation ( or control parameter) which can achieve the extremal value turned to hold the system at critical point. Such systems have attractor in the critical point. Some times the attractors can fluctuate creating the conditions of appearance of chaotic situations.

The chaotic regime of the dynamic systems in general is characterized by Tsuda (1992) with many factors. One of them is a strange attractor appearance. The strange attractor can be appeared changing a bifurcation parameter. It has a non-uniform character items from dynamic intermittent systems. The crucial property of the such behavior is interchange between two frequency (or two time scales) mode. We try search such properties in the immune systems like the neural systems by van der Mass et al. (1990), Garliauskas (1998). If to present the immune system as an immune network for appearance of the chaos, it is important to expose the conditions of the nonlinearities and an asymmetry of the network.

A simple immune network model with complex dynamics is analyzed by De Boer et al. (1990, 1993), the immune model with a Cayley tree by Anderson et al. (1993). The existence of chaotic behavior in an antibody/B cell (AB) model observed in (Stewart and Varela, 1990; Calenbuhr et al., 1995). Either cyclical or chaotic regimes were observed in host immune responses by Gupta et al. (1998). 


\section{Immune System}

It is well known that in the living being the humoral and cell-mediated immunity system are coexisted. The immunity response to the invaded antigen is the result of a complex interplay and mutual relationship of these two kinds of immunity with many components. The humoral immunity is based on $B$ lymphocytes. The cell-mediated immunity is based on $T$ lymphocytes, the $T$ procursors of which are produced in Thymus, including a cooperation between $T$ cells and macrophages. The antigen activated $T$ cells also produce clones of cells sensitive to the antigen through proliferation mechanism. One part of $T$-clone cells join the $B$ cells derived the memory other part join the activated macrophages overcomes the invaded antigen. These latter cells in contact with the target antigens synthesize and release substances called interleukins (IL-1, IL-2). Smith (1980) have suggested a participation of IL-1 and IL-2 in $T$ and $B$ of lymphocyte proliferation. Another function of interleukins is is the mobilization of the resting macrophages and their attraction to the invasion of the antigen and able to kill infected cells at a distance without intervention of macrophages. In addition, $T$ cells can attach to target cells (antigens) and kill them directly.

Main hard work on overcoming of an invaded virus $B$ lymphocytes carry out after antigen sensibility under $T$-helpers control on producing of the antibody clones. This is so called an humoral immune response (the left-site of Fig. 1).

Neutralization of antigens realizes under taking part of the plasma-cells, macrophages, cooperation with mature $T$ lymphocytes and lymphokines.

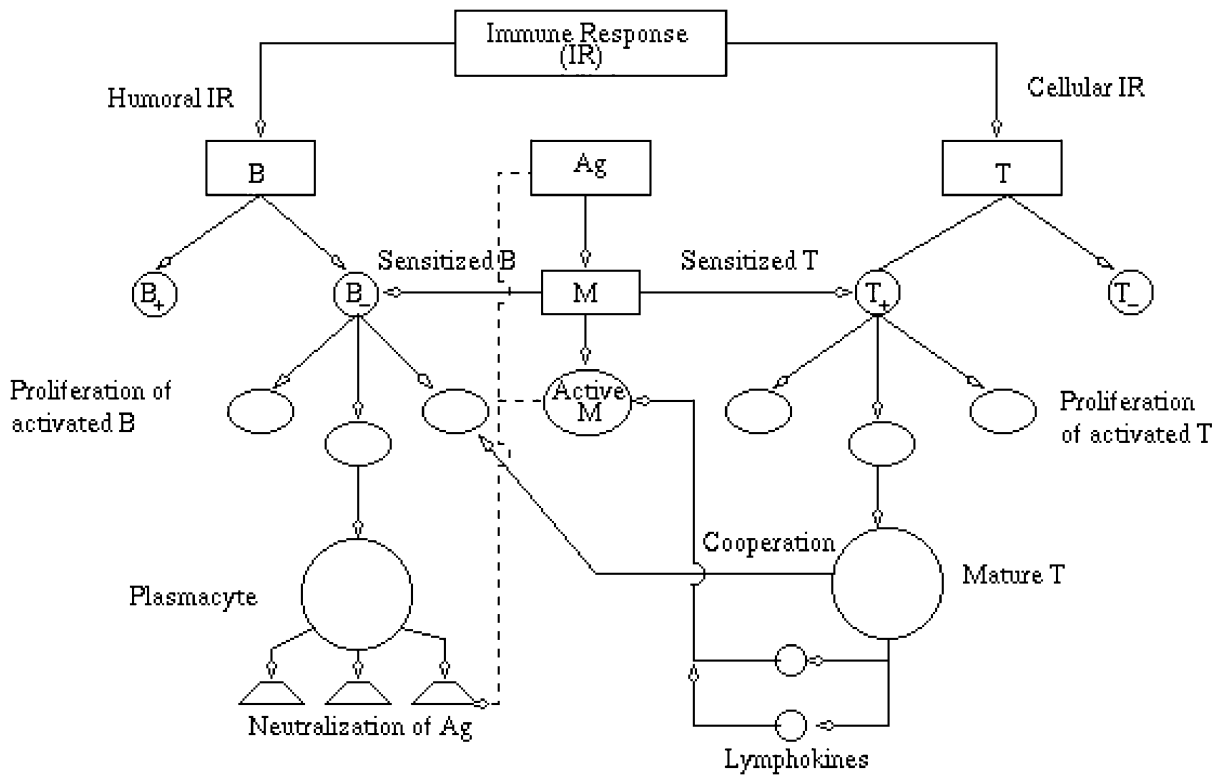

Fig. 1. The immune response simplified structure: $B_{+}, B_{-}$denote positive and idio-typical $B$ lymphocytes, $T_{+}, T_{-}$the same $T$ ones, $M$ is the macropfage, $A g$ denotes antigen. 


\section{Immune Network}

The model supposed by Bell (1970), describing the antibody production, explains high dose tolerance however this variant of model insufficient to describe a response to the high dose antigens, because no specific control mechanism. For the removing of this contradiction, Jerne (1974), Richter (1975), Hoffmann (1975) have introduced a limitation which is that the number of proliferation cells cannot be higher than some maximum level. Just mentioned authors with a help of the immune network network models with limitations overcome these lacks.

\subsection{Immune Network Prepositions}

According to the Jerne (1974) immune network theory is based on the following prepositions: (i) antibody and receptors of the lymphocytes can recognize and be recognized; (ii) lymphocytes ( $\mathrm{T}$ and $\mathrm{B}$ ) are able to the mutual action (synergism) and contraction (antagonism); (iii) antibodies of sensitized lymphocytes are able to response to recognizing signals as a positive or negative depending on different inner and outer conditions; (iv) self-behavior of the immune system is determined by interaction of lymphocytes and molecules of antibodies inside of the network recognizing each other.

Further we mostly follow (Hoffmann, 1975) where two sets of antigen-specific $T$ lymphocytes are presented for determination of the immune response to an antigenic stimulus. There are the antigen-binding $\mathrm{T}$ lymphocytes $\left(T_{+}\right)$and anti-idiotypic lymphocytes $\left(T_{-}\right)$, and also positively armed effector lymphocytes $\left(K_{+}\right)$(Fig. 2$)$. The $T_{-}$have receptors that are complementary to $T_{+}$and therefore they are not only stimulated by $T_{+}$ but can stimulate $T_{+}$in backwards. It means that both $T_{+}$and $T_{-}$have the same specificity of receptors. When the present achieves a high concentration, it inhibits mutual stimulation blocking the receptors of the lymphocytes.

It is important to emphasize a role of invoking a large number of different factors which allow to regulate of the immune response. As a rule, $T$ cell-selected immune factor is monovalent whereas $T$ receptor is at least divalent.

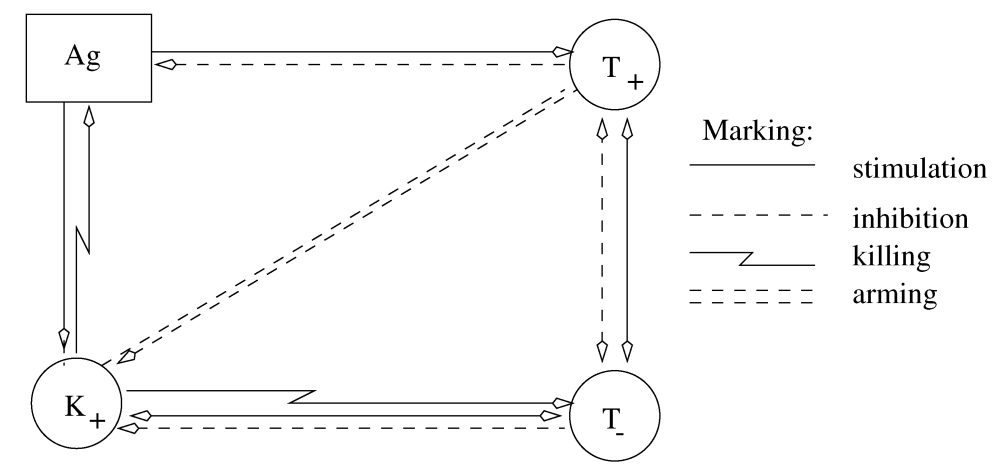

Fig. 2. The main complex of inter-linking antigen $(A g), T$ lymphocytes, and effector cells $\left(K_{+}\right)$. 
The cross-linking armed receptors and $T$ lymphocytes under an activation of antigen are schematically shown in Fig. 2.

The stimulation is denoted by solid lines. Blocking (inhibition) of receptors is represented by single dashed lines, and the killing of cells is shown by jagged lines. The armed effector cell is denoted by a double-dashed line.

Different types of the immune response are supposed to understand as collective phenomena defined by network structure which is represented by immune system itself in general.

\subsection{Immune Network Description}

According to Jerne (1974) and Richter (1975) the immune network is postulated by the qualitative properties: (i) antigen stimulates some class antibodies each of which represents an antigen for the same other class and so on; (ii) between input and inner epitopes and paratopes the relation stimulation (direct bind) and suppression (back bind) are existed; (iii) stimulation and suppression as well can be inhibited as a consequence of blocking paratopes and epitopes; (iv) it is necessary some saturation concentration of epitopes to include the immune-competent cell for differentiation.

The immune network presentation can be described in the following. The antigen $V^{(l-1)}$ represented as $l-1$ species stimulates the species $l$, i.e., positive $T_{i}^{l}$ lymphocytes (Fig. 3).

Latter activates the proliferation cells and producing of a clone of antibodies of $l$ species. The suppressive $T_{s}^{(l+1)}$ cells on the level of species $l+1$ suppress $T_{i}^{(l)}$. In parallel to $T_{s}^{(l+1)}$, the anti-idiotypical $T_{j}^{(l)}$ appear and inhibit (block) $T_{i}^{(l)}$. It activates and some times inhibit the effector cells $T_{e f f}^{(l)}$, and latter kill $V^{(l-1)}$. The killing process is carried out by antibodies $F^{(l)}$ jointly with macrophages. The antigen $V^{(l-1)}$ can indirect inhibit the species $T_{s}^{(l+1)}$. Besides suppression $T_{s}^{(l+1)}$ can become in the role of antigen and sensitize next block of immune network continuing net structure.

\section{Immune Network Chaos Model}

It is known from general dynamic system theory that under some defined circumstances the deterministic chaos is possible. This is confirmed that in the analogous to immune network there are the neural network systems according to the following works (Van der

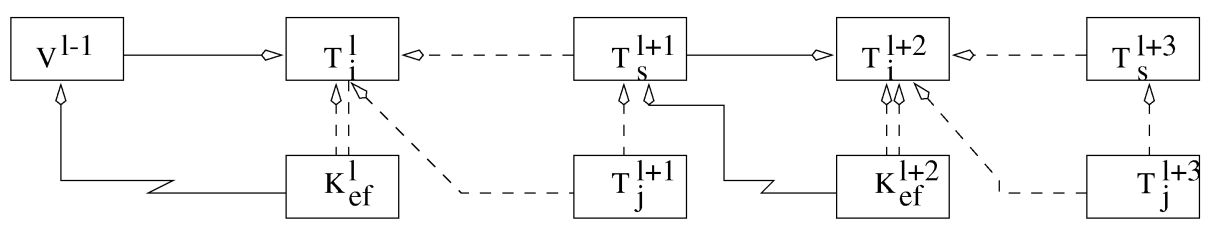

Fig. 3. The immune network system structure. 
Mass et al., 1990; Garliauskas, 1998). Below we will show that the immune networks are also able to the chaotic behavior under some prepositions.

\subsection{Immune Network Mathematical Model Description}

The basic assumptions to the postulate theory are the following. The triggering lymphocytes have the cross-linking receptors. The $T$ and $B$ lymphocyte receptors are immunoglobulin-like molecules. The cross-linking receptors lead to the activation of the cell-mediated macrophages armed with specific $T$ cell factors to eliminate cells of the complementary specificity.

Another part of the theory is that the cross-linking of receptors can be inhibited by blocking of the receptors by monovalent factors.

It is known from the theoretical morphogenesis that as the result of short range activation and long-range inhibition (by blocking) the stable dissipative structures, strange attractors and chaotic phenomenon can be formed. Mathematical modeling was constructed working out the ideas presented in this paper and on clonal approach by Bell (1970), network model by Richter (1975), Hoffmann (1975).

Discrete differential equations, limit conditions of concentration of immune values, cross-linking receptor assumptions, and two-mode ranging structure are the main base of the immune network modeling.

The basic regulation value of the immune response is the antigen. Its behavior is controlled by many factors such as an amount of specific cells, antibodies, sensitized macrophages, etc. For the description of the antigen concentration was used the following discrete differential equation:

$$
V(t+1)=V(t)+\alpha V-\beta V F
$$

where $\alpha$ is the difference between the growth rate and death rate of antigen, $F$ is the concentration of antibodies (they may be $T_{+}$or $T_{-}$), $\beta$ is the rate of specific killing by cytotoxic effector cells as a probability of antigen neutralization by the specific lymphocytes or antibodies at meeting with antigen, the growth of iteration parameter $t$ is equal to one.

Further, according to (Hoffmann, 1975) [12] and a little corrections, the antigenbinding $T$ lymphocyte concentration was described by discrete nonlinear differential equations as follows:

$$
T_{i}(t+1)=T_{i}(t)+T_{i}(t) R_{T(i, j)}^{i}-\sum_{l=1}^{n} \gamma\left(V_{l}(t) T_{i l}+I_{T_{i} l}\right.
$$

where $T_{1}$ denotes $T_{+}, R_{T(i, j)}^{i}$ is the rate of replication $T_{+}$, due to stimulation by type $j$, $\gamma\left(V_{l}(t)\right.$ is the function of $l$ antigen at $t$ moment under influence by limited function $\gamma\left(V_{l}(t)=a_{1} V_{l}(t) /\left(b_{1}+V_{l}^{m}(t)\right)\right.$, where it at $V_{l}(t) \rightarrow 0$ limits to zero and at $V_{l}(t) \rightarrow \infty$, $m=1$ reaches a saturation expressed by $a_{1}, b_{1}$ is a fixed value to prevent calculations 
from an uncertainty, and $n$ is the number of antigens. Further, $T_{i l}$ is $T_{+}$specified to 1 antigen in the network, $I_{T_{i} l}$ is the rate of immigration of $T$ lymphocytes of the same specificity from stem cells, and the cells after mutation with other specificities. Eq. (1) is used the same for different antigens.

The rate of replication $T_{i l}$ will have a complex structure of interacted parameters and can be expressed

$$
R_{T(i, j)}^{i}=\frac{1}{\tau_{R T}} \frac{c_{1}}{1+V(t)+\left(T_{j} e_{R T}\right)^{m_{1}}}
$$

where $\tau_{R T}$ is the replication rate constant, $c_{1}$ is a saturation of concentration $T_{i l}, m_{1}$ is the grade characterizing the with of thresholds, and variable $e_{R T}$ is s the effectiveness of collision between $T_{i l}$ and $T_{j l}$ in triggering of $T$ cells. This effectiveness could be blocked by $T$ cell factors.

Thus, the effectiveness of collisions can be expressed by dependence

$$
E_{R T}=\frac{C_{b l} T_{i}}{1+T_{i}^{m_{b l}}} \frac{C_{b l} T_{j}}{1+T_{j}^{m_{b l}}},
$$

where $C_{b l}$ denotes the threshold of concentrations of $T$ factors for blocking $T$ cells, and $m_{b l}$ is the with of the threshold. The collision effectiveness (3) is the product of two terms defined by $T_{i}$ and $T_{j}$ cells.

The suppressed lymphocyte concentration rate is described by equation

$$
T_{s}(t+1)=\left(1-D_{s}\right) T_{s}(t)+\sigma T_{s}(t)
$$

where $D_{s}$ is death rate of $T_{s}$ cells, $\sigma$ denotes the growth rate of the suppressed cells at $t$ moment.

The $T_{-}, B_{+}$and $B_{-}$concentration rates in dynamics can be described by the analogous to Eqs. (2)-(5).

The recursion Eqs. (1), (3) and (5) including expressions (3) and (4) have been solved until the saturation state would be achieved. The iterations are stopped when the convergence error exceeds the given value. This decision procedure belongs to a short-range activating iterations with the cross-linking of receptors.

The antibody recursion equation may be represented as follows:

$$
\begin{aligned}
F(k+1)= & \left(1-D_{F}\right) F(k)+C(k)-\theta a_{2} V(t+1) F(k) \\
& -\eta a_{3} T_{s}(t+1) T_{i}(t+1),
\end{aligned}
$$

where $D_{F}$ is the death rate of the antibody concentration, $C(k)=\rho F(k) V(t+1)$ is the plasma cell concentration rate which is proportional to the antigen-antibody complex value, $\theta$ is the coefficient belonging to probability of neutralization antigen by antibody at their collision, $a_{2}$ is the number of antibodies for neutralization of one antigen at time unit. The coefficient $\theta$ was used as a control or bifurcation searching the chaotic phenomena of the immune network. The recursion Eq. (6) reflects the slow process equation 
on each step of which the quick Eqs. (1), (2) and (5) are solved until stationary states achieved.

\subsection{General Prepositions to Chaotic Behavior}

The dynamic systems especially dissipative dynamics systems are able to have solutions that at $t \rightarrow \infty$ attract to some subset measure zero in phase space. The chaotic oscillations in the dynamic systems characterize by a strange attractor. The strange attractor structure is more complex than the usual attractor. It is able to form a geometrical invariant structure or named a scaling structure. If the dynamic system dependents on a parameter, its changing can challenge a qualitative variation of the attractor. Such value of the control parameter is the bifurcation value or the bifurcation. In this case it can happened a period-doubling process continued until the endlessness. It is known that the Feigenbaum universal constant achieves itself defined value $(\delta=4.6692 \ldots)$ independent of the system type, choosing of representation, and a scale of system.

The systems in more general position can be characterized by two timescales (quickly and slowly), dynamic, heterogeneous, unstructured, open networks:

- timescales: when one part system is carried out quickly and another one is worked slowly;

- dynamics: when the states are far from equilibrium governed by attractors and trajectories;

- heterogeneous: when the modes of the network and the connections among elements can be of many different types;

- unstructured: there is no regularity in the network connectivity;

- ppen: the components are not fixed, new kinds of nodes and connections may appear.

The vertebrate immunological systems detecting and preventing security attacks of invaded antigens have such properties.

The immune system represented by nonlinear dynamic equations can give rise to the chaotic behavior as we show by computational experiment in the next section.

\subsection{Computational Experiments}

The computational experiments based on two-dimensional immune antibody/B cell (AB) models with task to analyze the dynamical features were carried out in (De Boir et al., 1990; 1993). However, the AB model is nonrealistic because of in AB an antigen is not included. It is known that without antigen the cell differentiation and proliferation a production of antibodies is not possible. In spite of this model even in a simplified form have allowed to observe the complex dynamical behavior including either stationary, periodic or apparently chaotic regimes. The analogous chaos, persistence, and evolution of strain structures were modeled in (Gupta et al., 1998; Andersen et al., 1993; Calenbuhr et al., 1995) and others.

In our case simulation of the dynamic immune network processes has been carried out in two iteration stages. In first stage, the discrete differential Eqs. (1), (2), and (5) regard- 
ing to expressions (3) and (4) were calculated by recursion procedure until the convergence criterion exceeds the given value $\left(\Delta T_{i}=0.02\right)$. After that the second calculation stage is switched on. The first step of recursion (6) is solved at zero initial conditions and using last values of the positive lymphocyte $T_{i}$, suppression lymphocyte $\left(T_{s}\right)$, and antigen concentrations of quick cycle iterations. The slow and quick cycles continue until the stationary state of the antibody concentration rate will be achieved. After that the coefficient $\mu=\eta a_{2}$, as an average of number of antibodies for killing antigen will be changed with a small growing of $\mu(\Delta \mu=0.01)$. This coefficient, if the dynamic solutions bifurcate, is named a bifurcation parameter.

Investigation of chaotic behavior effects in the immune network system occurred by registering the sum of absolute values of the antibody concentrations for different values of $\mu$.

For an illustration, there was used the three block-unit immune network where the immigration $T_{i}$ lymphocyte concentration rates $I^{l}, I^{l+1}, I^{l+2}$ were given. The coefficients $\alpha=0.1, \beta=0.5, a_{1}=0.5, b_{1}=1.5, a_{2}=2.5, c_{1}=10, \tau_{R T}=100, m=2, m_{1}=2$, $m_{b l}=2, C_{b l}=100, D_{s}=0.1, \sigma=0.3, D_{F}=0,1, \rho=0.1$, and $\theta=0.4$ were fixed as conditional ones. The absolute sum of antibody concentrations was defined after the value of recursion was stabilized from 149 to 210 cycles.

The first diagram in Fig. 4 characterize the multi-macrostate regimes in the immune network system. These regimes with the immunological interpretation can be characterized in such a way. The concentration of antibodies in the beginning a little decays and

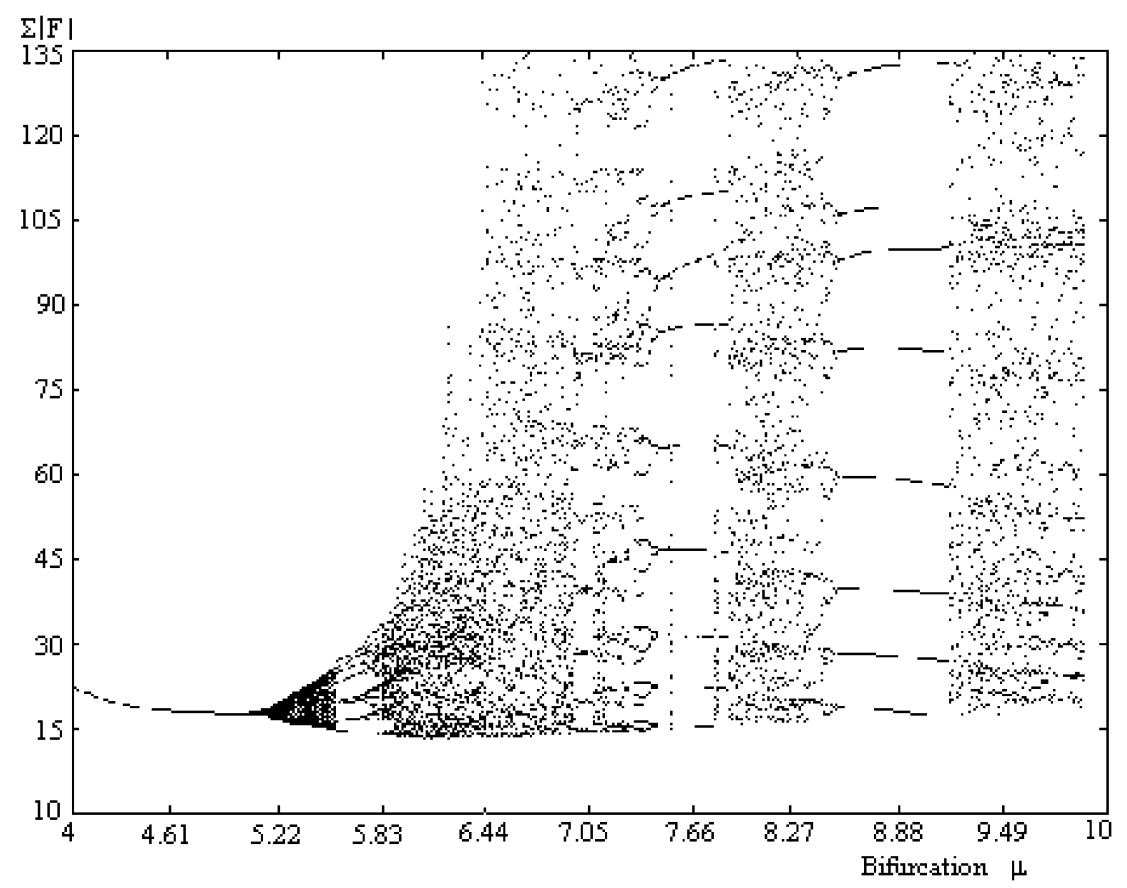

Fig. 4. The diagram of the immune network regime. 
further becomes stable until no invasion of antigen. Around $\mu=5.2$ the bifurcation appear. The antigen attacks the organism and activates $T$ and $B$ lymphocytes. The proliferation and differentiation mechanisms are switched on. Apwards values $\mu=5.83$ till $\mu=6.95$ the system reaches chaotic reproduction of antibodies forming an appropriate clone. There also are seen the periodic windows at $\mu=5.52, \mu=7.35$, and $\mu=8.87$ which may be interpreted as a memorization of high sensitive lymphocytes.

The other diagram in Fig. 5 is showing almost similar behavior but without clear expressed windows. Here the calculations were made with changing of immigration values $T^{l}=T^{l+1}=I^{l+2}=10$ and steps of the iterations $\Delta T_{i}=0.2$, and recursion $\Delta \mu=0.7$. The complex processes are now observed in another range of bifurcation parameter. Here it has been the regular range $(\mu=50 \div 100)$, bifurcation of solutions $(\mu=110 \div 130)$, windows of periodical $(\mu=130 \div 140)$ and chaotic behavior (after $\mu=140$ ). The immunological interpretation is analogous to the first diagram.

Though the meager experimental reports suggesting that concentrations of idiotype and anti-idiotype pairs distribute in an irregular and possibly chaotic, to confirm complete conclusion of an existence of chaos in the immune response so far is not possible.

Suppose that for experimental immunology will be much hard problem to prove such complicated behavior in the immune network dynamic system.

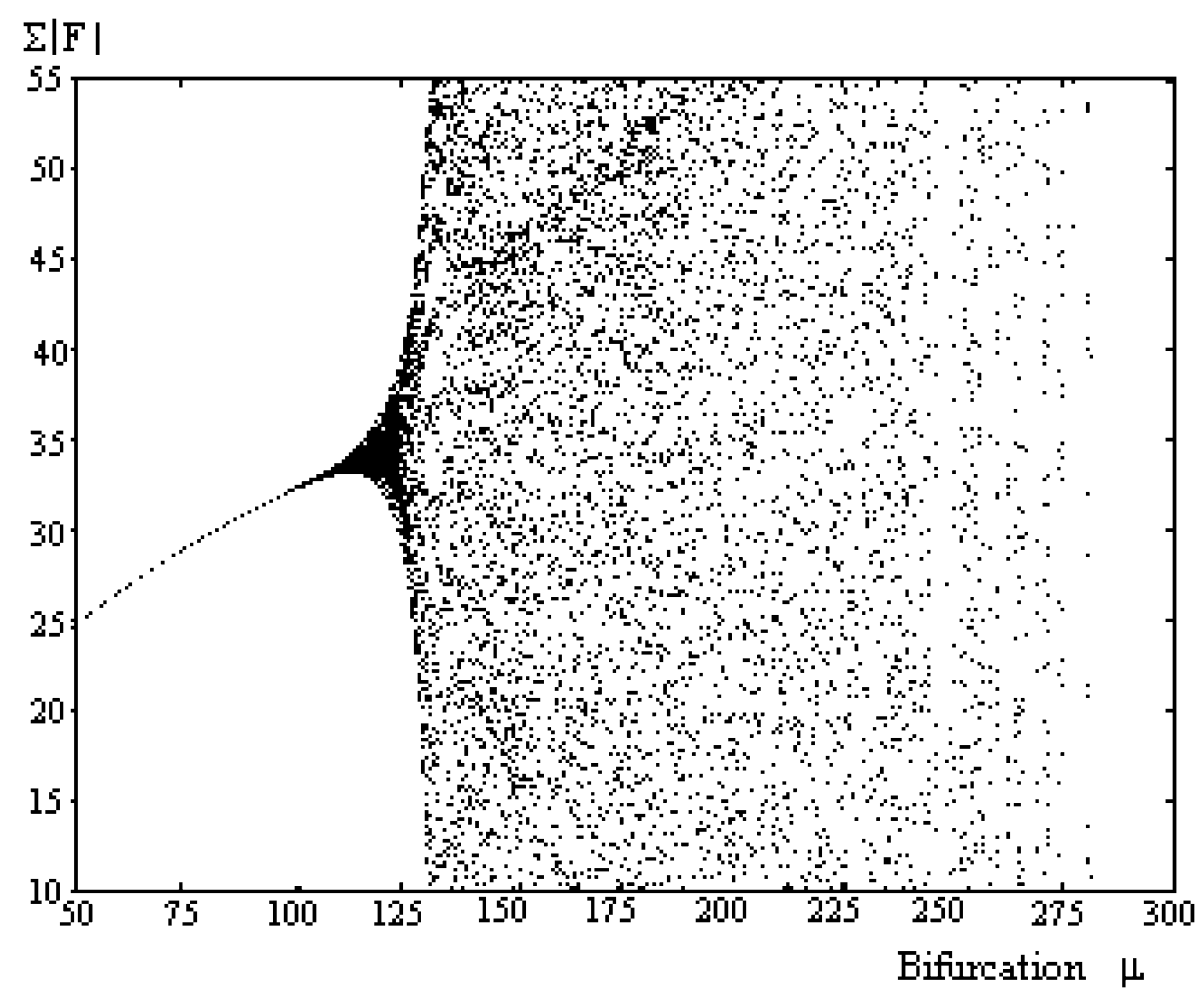

Fig. 5. The diagram of the immune network regime after changing of parameters. 


\section{Conclusions}

1. The immune network system was presented by the sequence of species with new immunological components allowing more plausible to reflect the immune response processes.

2. An hypothesis of the chaotic behavior in the immune network system is formulated from theoretical and computational experiment positions.

3. The mathematical model oriented to the describing of the more realistic immune processes in the dynamics allowed to organize the computational experiment in the first to demonstrate a possible very complex behavior including chaotic regimes.

\section{References}

Andersen, R.W., A. Neuman, A.S. Perelson (1993). A Cayley tree immune network model with antibody dynamics. Bull. Math. Biol., 55, 1091-1131.

Bell, G. (1970). Mathematical model of clonal selection and antibody production. J. Theor. Biol., 29, 181-232.

Bell, G. (1971). Mathematical model of clonal selection and antibody production, II. J. Theor. Biol., 33, 339378.

Bell, G. (1973). Predator-prey equations simulating an immune response. Math. Biosci., 16, 291-314.

Burnet, F.M. (1959). The Clonal Selection Theory of Acquired Immunity. Cambridge University Press, Cambridge.

Calenbuhr, V., H. Bersini, J. Stewart and F.J. Varela (1995). ??? Straipsnio pavadinimas ??? J. Theor. Biol., 147, 776-786.

De Boir, R.J., I.G. Kervekidis and A.S. Perelson (1990). A simple idiotypic network model with complex dynamics. Chem. Eng. Sci., 45, 2375-2382.

De Boir, R.J., A.S. Perelson and I.G. Kervekidis (1993). Immune network behavior - II. From oscillations to chaos and stationary states. Bull. Math. Biol., 55, 781-816.

Garliauskas, A. (1998). Neural network chaos analysis. Nonlinear Anal., Model. and Control, 3, $43-57$.

Gupta, S., N. Ferguson and R. Anderson (1998). Chaos, persistence, and evolution of strain structure in antigenically diverse infections agents. Science, 280(5365), 912-915.

Hege, J.S., and G. Cole (1966). A mathematical model reflecting circulating antibody and antibody forming. $J$. Immunol., 97, 34-40.

Hoffmann, G.W. (1975). A theory of regulation and self-nonself discrimination in an immune network. Eur. J. of Immunol., 5, 638-647.

Jerne, N.K. (1974). Towards a network theory of the immune system. Ann. Immunol., 125C, 373-389.

Jilek, M. (1971a). The number of immunologically activated cells after repeated immunization (a mathematical model). Folia Microbiol., 16, 12-23.

Jilek, M. (1971b). On contact of immunocompetent cells with antigen (Note on probability model). Folia Microbiol., 16, 83-87.

Marchuk, G.I. (1978). Some mathematical models in immunology. In Proc. of the 8th IFIP Conference on Optimization Techniques. Springer-Verlag, Heidelberg. pp. 41-62.

Marchuk, G.I. (1980). Mathematical Models in Immunology. Nauka, Moscow (in Russian).

Mohler, R., et al. (1978). T- and B-cell models in the immune system. Theoretical Immunology, Marcel Dakker 23.

Nicolis, G., and I. Prigogin (1979). Self-Organization in Non-Equilibrium Systems. Mir, Moscow (in Russian). Prikrilova, D. et al. (1984). A model of proliferation control in immune response. Kibernetika, 4(1), 37-46.

Richter, P. (1975). A network theory of the immune system. Eur. J. of Immunol., 5, 350-354.

Smith, K.A. (1980). T-cell growth factor. Immunological Review, 51, 337-357.

Stepney, S. (2003). Critical critical systems. In LNCS, vol. 2629. Springer. pp. 62-70.

Stewwart, J., and F.J. Varela (1990). ??? Straipsnio pavadinimas ??? J. Theor. Biol., 153, 477-498.

Tsuda, I. (1992). Dynamic link of memory - chaotic memory map in non-equilibrium neural networks. Neural Networks, 5, 313-326. 
Van der Mass, M.L.J., P.F.M.J. Vershure and P.C.M. Molenaar (1990). A note on chaotic behavior in simple neural networks. Neural Networks, 3, 119-122.

A. Garliauskas received his habil. dr. degree of technical sciences from the Computer Center, the Department of the USSR Academy of Sciences, Novosibirsk, USSR, in 1977. $\mathrm{He}$ is a head of the Laboratory of Neuroinformatics, Institute of Mathematics and Informatics and a professor in Vilnius Gediminas Technical University. His research interest includes neuroinformatics methodology, control problems and development of neural networks algorithms, chaos processes.

\section{Imuninio tinklo sistema su chaotiniu elgesiu}

\section{Algis GARLIAUSKAS}

Imuninio tinklo sistema pavaizduota kaip elementų seka su naujomis imunologinėmis komponentėmis, leidžiančiomis kuo adekvačiau atspindèti imuninio atsako procesus. Tokios sistemos chaotinis elgesys yra formuluojamas remiantis teorinèmis bei skaičiavimo eksperimento pozicijomis. Pateikti imuninès sistemos chaotinio elgesio modeliavimo rezultatai. 